\title{
Erratum
}

\section{A Facile Access to Antiflu Agent Tamiflu/Oseltamivir}

Hai Sun, Ying-Jie Lin, Yu-Lin Wu,* Yikang Wu* Synlett 2009, 2473.

In the original Scheme 3 the configurations of compounds 10, 19 and $\mathbf{2 0}$ were incorrect. The correct Scheme is shown below. Further, the Supporting Information file has been replaced. The authors apologize for this mistake.

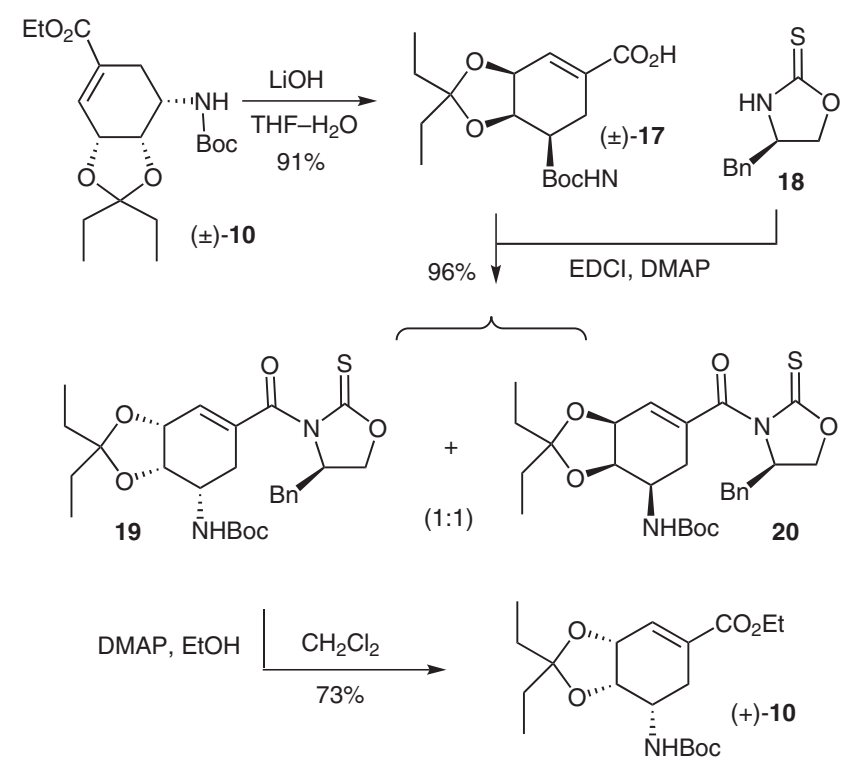

Scheme 3 